Voix et Images

voixetimages

\title{
Les avatars de l'histoire : Les Grands-pères de Victor-Lévy Beaulieu
}

\section{Jacques Michon}

Volume 5, numéro 2, hiver 1980

Yves Thériault

URI : https://id.erudit.org/iderudit/200208ar

DOI : https://doi.org/10.7202/200208ar

Aller au sommaire du numéro

Éditeur(s)

Les Presses de l'Université du Québec

\section{ISSN}

0318-9201 (imprimé)

1705-933X (numérique)

Découvrir la revue

Citer cet article

Michon, J. (1980). Les avatars de l'histoire : Les Grands-pères de Victor-Lévy

Beaulieu. Voix et Images, 5(2), 307-317. https://doi.org/10.7202/200208ar d'utilisation que vous pouvez consulter en ligne.

https://apropos.erudit.org/fr/usagers/politique-dutilisation/ 


\section{Les avatars de l'histoire: \\ Les Grands-pères de Victor-Lévy Beaulieu}

A l'instar du nouveau roman, le récit de Victor-Lévy Beaulieu ne détruit pas l'esthétique réaliste mais en perpétue et renouvelle la fonction. Tout en préservant les catégories admises du genre, comme le personnage, l'anecdote. Beaulieu remet en question dans certains de ses textes la pertinence d'une histoire chronologique et progressive. Pour lui la narration traditionnelle n'est pas assez fidèle au réel et lait illusion, il définit l'histoire comme " un cataloguage (sic) d'événements arbitraire que, par après, on rend logiques, les enchainant les uns aux autres pour donner l'illusion d'une homogénéité -qui n'a jamais existè »'. A cette fausse cohérence ou cette élaboration secondaire qui vise à rendre logique un enchainement hétérogène d'événements, le romancier va substituer une représentation fragmentée de la réalité: " il n'y a qu'une caractéristique dans l'histoire, qui vaut aussi pour l'individu; it n'y a que des fragments parce que toute vie est fragmentaire, faite de coincidences sérielles, absolument réfractaires à toute rationalité $*$ (Ibid.).

Ce parti pris a néo-réaliste * n'est pas visible au même degré dans tous les récits de Beaulieu. Certains romans comme Race de monde (1969) et Jos Connaissant (1970) reproduisent les structures du journal ou du récit autobiographique, alors que Don Quichotte de la démanche (1974) et Un rêve québécois (1972) déconstruisent le discours narratif classique. Pour rendre compte de cette diversité et illustrer ce qu'on pourrait appeler la visée antidiégétique du récit de Beaulieu, nous examinerons Les grands-pères (1972), roman qui semble occuper un lieu intermédiaire dans la production de l'auteur entre la tradition et la modernité. Ce récit nous permettra de cerner les effets et les limites de ce travail de déconstruction. Pour ce faire nous utiliserons l'analyse de la temporalité discursive mise au point par Gérard Genette dans Figures $1 / 2$ et qui repose sur l'opposition classique discours/histoire ${ }^{3}$. En nous arrêtant surtout aux figures d'ordre, nous aurons l'occasion de vérifier comment ce rapport dialectique s'effectue et au profit de quelles catégories esthétiques.

La confrontation de l'ordre des événements dans le récit et dans l'histoire va nous fournir un premier découpage du texte. Si l'histoire est 
toujours chronologique et irréversible, le récit bouleverse l'ordre des événements pour les faire signifier à un deuxième degré. Ainsi dans Les grandspères l'hịstoire de Milien nous est racontée de deux manières différentes; par un récit de première instance et par une série de retours en arrière (analepses) qui récupèrent le passé du héros. Les fonctions de ces deux niveaux semblent à priori conventionnelles: le récit premier expose et décrit le parcours du héros dans son village natal et les analepses viennent bloquer et arrèter l'action en cours, en relarder le développement, pour faire place aux souvenirs qui envahissent la conscience du vieillard. Cette dialectique entre les deux niveaux de fiction paraît simple. jusqu'au moment où l'attente créee depuis le début du récil et entretenue par de nombreuses prolepses se trouve à la lin déçue, déjouée.

Résumons le récit premier pour illustrer ce parcours: Milienne II, la seconde femme de Milien, termine la vaisselle puis s'affaisse sur le plancher de la cuisine. Est-elle morte? Milien sort de la maison pour chercher de l'aide. Mais le vieillard perd la mémoire, oublie le but de sa sortie et finalement erre tout l'après-midi dans le village: il va prier à l'église pour sa fille Mathilde; il se promène dans le cimetière; surpris par une tempête, il se réfugie au magasin gènéral où il joue aux cartes avec des compères (les Milien, les grandspères) ; il rend visite a son ami Chien Chien. Puis a la fin de la journée il rentre chez lui. Est-ce pour venir en aide (enfin!) à Milienne? Non, Milien rencontre le médecin qu'il devait aller chercher; la maison est vide et semble avoir été abandonnée depuis longtemps. Le médecin ausculte le vieillard, le lave, le couche et le laisse seul aux prises avec la mort qui approche.

Lorsque le récit se termine le lecteur se demande ce qui a bien pu advenir de Milienne, abandonnée au début du récit. D'autant plus qu'à plusieurs reprises Milien affirme la nécessité de retourner à la maison pour venir au secours de sa femme:

«Faudrait que je parte». Et: «Milienne m'attend. (p. 74) II fallait rentrer à tout prix maintenant : quelqu'un mourait dans sa maison, qui n'avait encore besoin de lui que pour partir selon les formes. (p. 133).

[...] il faudrait bien prévenir le médecin que Milienne s'en allait. (p. 148).

Or lous ces énoncés qui créent une attente chez le lecteur, attente de voir Milien venir au secours de Milienne ou de savoir enfin ce qui est arrivé à celleci durant les tribulations de son mari, sont des leurres. D'après l'ètat pitoyable de la maison et l'attitude du médecin il apparait que Milienne était morte depuis déjà un certain temps, c'est-à-dire bien avant que le récit ne commence.

Nous sommes donc invités à relire le premier chapitre (l'évanouissement de Milienne) non plus comme un fait rèel du récit premier mais comme un souvenir ou un rève du vieillard. La quète de secours devient donc une lausse quête, un leurre entretenu par Milien pour masquer sa propre mort. La vraie quête de Milien est ailleurs. Ainsi le lecteur se rend comple que lout au long du récit il a élé trompé par le héros et son narrateur, qu'il a été 
lancé sur une fausse piste et que le sens du récit jouail ailleurs, non dans le présent mais dans le mouvement qui va du présent au passé. La forme analeptique du récit indique bien là où il faut lire la quête de Milien.

La fin de I'histoire nous renvoie à l'univers onirique de Milien qui constitue le second niveau du récit. En effet au cours de ses tribulations a travers le village, Milien perd constamment le fil de sa "fausse quête"; sous l'effet du gin ou du sommeil il réve et revoit son passé. Ces visions rétrospectives se suivent pêle-mêle, sans ordre chronologique. Si l'on tente de meltre un peu d'ordre dans cette suite anachronique d'événements on arrive a déterminer trois grandes positions temporelles dans la vie antérieure du héros:

1. l'enfance,

2. la vie avec Milienne I.

3. la vie avec Milienne II.

La seconde période se dịvise elle-même en deux parties:

2 a. Ia vie heureuse avec Milienne I.

b. Ia maladie et l'agonie de Milienne I.

Cette seconde division est fondamentale dans l'histoire, en fait elle partage l'univers de Milien en deux phases: d'une part le versant euphorique représenté par les scènes de l'entance et de la vie heureuse avec Milienne I, d'autre part le versant dysphorique. la dislocation du monde de Milien qui commence avec la maladie et la mort de Milienne I. Cette rupture est signalé à plusieurs reprises (voir pp. 76, 145-146). Le début de la maladie de Milienne I represente le point de non-retour, la rupture irréversible par rapport à un équilibre initial. On pourrait illustrer la diégése comme suit:

Equilibre (1. et $2 a) \rightarrow \longrightarrow$ Déséquilibre $(2 b$ et 3 ).

Lorsque le récit commence le héros n'a pas réussi a surmonter son aliénation et semble y avoir renoncé. La position temporelle 3 (le remariage) représente la derniere tentative de Milien pour retrouver le temps perdu. Quelque chose s'est brisé avec la Maladie de Milienne I qui ne peut plus être réparé; ne reste plus que le rappel de la vie passèe par le souvenir et le réve. La seule transformation, le seule changement dans l'histoire de Milien a eu lieu avant le début du récit. Lorsque le héros entre en scéne les jeux sont faits, le sort en est jeté: Milien doit mourir sans pouvoir retrouver le bonheur perdu. Le récit est un lieu déjà dévasté par la mort de (ou des) Milienne. Milien sait qu'il va mourir, toutes les prolepses (segments narratifs d'anticipation) laissent planer cette menace (voir pp. 27, 45-46, 132, 157), et la scène antérieure indépassable (la mort de Milienne l) bloque toute évolution possible du héros.

La reconstitution de l'histoire se fait donc en deux termes; le sens de I'histoire n'est pas une donnée immédiate du récit, il est déduit a posteriori après le détour d'une première lecture. Le récit est encadré par deux fonc- 
tions solidaires, l'aller/le retour; entre ces deux fonctions limites les actions s'organisent surtout à partir des lieux, (la géographie du village: l'église, le cimelière, le magasin général) comme dans un récit épique. Si le départ implique le retour, ce retour prévu et annoncé tout au long du récit ne se lait pas là où on l'attendait. Le récit mime en parcours pour ensuite l'invalider et nous inviter à lire ailleurs son sens, c'est-à-dire dans la construction du roman caractérisé par la prolifération des analepses.

L'analepse occupe une position centrale dans Les grands-pères, elle joue un róle important dans la production du sens. Le récit rétrospectif tient une place et une fonction qui le distingue d'une narration traditionnelle. Nous allons tenter d'indiquer la portée et le sens de celte construction.

Sur le plan de la fréquence d'abord: en établissant la quantité des segments rétrospectifs par rapport aux segments du récit premier, nous arrivons aux données suivantes:

\section{Niveaux}

Longueur

Recit premier (l'après-midi de Milien) .......... $39 \%$

Analepses (vrais ou faux souvenirs de Milien) .... $61 \%$

Ensemble du recit ..................... 100\%

On constate que le récit analeptique dépasse en longueur le récit premier: dans l'ensemble du roman six pages sur dix sont consacrées a la rétrospection. Proportion impressionnante si l'on considere que dans le récit traditionnel les analepses sont toujours secondaires, ayant d'abord une fonction explicative et complémentaire, alors qu'ici le rapport semble inversé. Le récit premier a la fonction d'un récit cadre, il sert de prétexte à une enfilade d'épisodes rétrospectifs. Mais bien qu'il soit au second rang sur le plan quantitatif, on ne doit pas oublier qu'il joue un rôle idéologique important puisqu'il permet de lire encore ce roman selon les règles de la représentation classique.

Dans l'œuvre de Beaulieu Les grands-pères semble occuper sur ce plan une position intermédiaire entre ce qu'on pourrait appeler les récits oniriques (circulaires et achroniques) et les récits historiques (linéaires et chronologiques). En effet dans certains romans comme La nuilfe de Malcomm Hudd (1969). Un rêve québécois (1972) le fil de l'histoire est rompu, le code herméneulique défait, de telle sorte qu'il n'est guère possible de reconstituer I'histoire "vraie" du récit. Dans d'autres romans comme Race de monde (1969), Jos Connaissant (1970), le récit est construit selon un plan beaucoup plus conventionnel. Les grands-pères se situe à mi-chemin entre ces deux póles.

Si on s'arrète à la distribution des analepses dans le récit, on constate que, contrairement au discours romanesque traditionnel qui concentre les 
analepses dans la première partie du récits, dans Les grands-pères les segments iétrospectifs sont étalés sur toute la longueur du texte. Cette construction en zigzag a pour résultat d'enliser davantage l'évolution de l'histoire d'empêcher toute progression continue de la diégèse initiale. L'analepse vient bloquer le déroulement du récit comme les souvenirs viennent bloquer toute progression possible du héros.

Cet enlisement du récit dans le passé peut être illustré par l'emboitement des analepses. Les segments rétrospectifs s'enchássent les uns dans les autres jusqu'au troisième degré, et ces analepses gigognes se suivent sans revenir nécessairement au premier niveau. Prenons par exemple le déroulement du chapitre 9 (p. 82-92) qui commence sur une analepse greffée sur l'épisode de la partie de cartes (les chiffres entre parentheses indiquent les pages):
1. récit premier
2. analepses
-3. analepses sur analepses
Milien joue aux cartes Agonie de Milienne_. Milien fait boucherie (86) (73) .. (83)
Milien perd une (87)
partie (92)

Ce parcours qui ouvre sans cesse de nouvelles parenthèses rétrospectives à l'intérieur des séquences-souvenirs, et que l'on pourrait représenter de la façon suivante 1 [2 (3) 2 (3)] 1, est assez représentatif de l'ensemble du texte qui ne revient au premier niveau que pour s'enliser encore dans une série de segments rétrospectifs.

En entrant en concurrence avec le récit premier l'analepse ne fait pas qu'arréter la progression de la diégèse principale, elle tend aussi à se substituer à elle, à prendre sa place. L'analepse dans Les grands-pères n'obéit pas à la règle énoncée par Genette (F III, p. 102) qui veut que le récit analeptique s'interrompe franchement sur une ellipse et que le récit reprenne là où il s'était arrêté. Lorsque la rétrospection est acheveee, on se trouve à un point ultérieur du récit. Pendant que te récit rétrospectif se déroule, le temps de l'histoire continue à courir lui aussi parallèlement; de sorte que le temps rétrospectif au lieu de venir s'ajouter à l'histoire pour la compléter, se substitue à elle; le temps onirique fait partie du temps de l'histoire. Ainsi pendant que Milien joue aux cartes avec les Milien (champ. 6 a 10) on est plongé dans une série de scènes rétrospectives qui évoquent la vie avec Milienne I: le temps qu'occupe la narration de ces épisodes c'est le temps de la partie de cartes elle-même. Lorsque le lecteur revient au récit premier il constate que la partie est déjà avancée, que la durée subjective a été substituée a la durée objective du récit ${ }^{6}$. Dans le récit traditionnel, l'analepse externe partielle maintient el préserve l'intégrité de l'histoire, ici, au contraire, le temps onirique de Milien envahit le réel, se substitue a lui; de telle sorte 
que le récit premier n'est plus qu'un fil conducteur de rêves et de fantasmes, qu'un support de lecture, qui d'ailleurs disparait dans certains romans de Beaulieu comme nous l'avons déjà noté. La représentation classique de I'histoire tend à disparaittre au profit d'un espace onirique, anachronique et ambigu. Le Don Quichotte de la démanche représente sans doute l'aboutissement de cette démarche.

II faut souligner une autre particularité importante des analepses dans ce récit. Presque toutes les anachronies relevées sont externes et partielles. Elles sont externes en ce sens que tous les episodes qu'elles relatent sont antérieurs au début du récit ', et elles sont partielles parce que ces épisodes du passé ne viennent jamais rejoindre le recit premier ${ }^{\circ}$. Autrement dit, il y a des trous dans l'histoire qu'aucun retour en arriere ne vient combler. Ce qui frappe ici c'est l'uniformité et la fréquence du procédé. Comme l'évocation de la vie passée occupe une place trés importante dans Les grands-peres, il est étonnant de voir qu'it n'est jamais possible de combler l'ellipse temporelle qui sépare le passé du présent. En fait cette impossible fermeture ou bouclage de l'histoire, dont les anachronies rendent compte ici, nous renvoient directement à une autre question de rhétorique narrative: la focalisation.

Cetle construction est motivée et vraisemblabilisée par une focalisation interne où le point de vue du narrateur se confond presque toujours avec celui du héros. Les événements sont décrits la plupart du temps comme s'ils étaient vus par le héros principal; l'organisation temporelle du récit (l'histoire fragmentaire) peut donc être mise au compte de la psychologie du vieillard. Si l'histoire est toujours incomplete et lacunaire, c'est parce que Milien la vit de cette manière, que Milien a des trous de mémoire, qu'il ne parvient plus á se remémorer sa vie antérieure, a la recomposer:

[...] sa mémoire était maintenant une espéce de puits dans lequel il n'avait plus le courage de descendre pour calfeutrer les fissures; il fallait laisser luir l'eau désormais, ne pas l'emprisonner; elle allait trouver son chemin toute seule, et peut-être méme reviendrait-elle dans le noir de sa mémoire comme cela arrivait parfois pour son passé dont les fragments lui rappelaient qu'il avait bien eu lieu, qu'it avait été lourd et gras, mais qu'il était trop tard pour le recomposer en son entier. (p. 39).

La juxtaposition de ces éléments hétérogènes, que sont les analepses externes partielles, est donc motivée el vraisemblabilisée par un argument psychologique. On retrouve ici la visée néo-réaliste des romans de Beaulieu.

La modernité de l'écriture beaulieusienne est produite surtout par l'usage de procédés introduisant une certaine ambiguité dans l'histoire. En utilisant l'homonymie, en gommant certaines transitions stratégiques entre séquences oniriques el séquences du réel et en évitant presque systématiquement le sommaire, le récit mel en cause la cohérence (la noncontradiction) sur laquelle le romancier ordinairement s'appuie pour faire illusion et créer l'effet de réel. 
Par exemple le fait d'attribuer à un nom de personnage ou de lieu des contenus diftérents ou opposés contribue à jeter une certaine confusion dans le récit et à mettre en doute son caractère véridique. Dans Les grands-pères cette confusion porte sur le nom de Milienne qui recouvre des contenus opposés:

\begin{tabular}{|c|c|c|}
\hline Signifian & \multicolumn{2}{|c|}{ MILIENNE } \\
\hline Qualifications & $\begin{array}{l}\text { Première femme de Milien } \\
\text { (63) } \\
\text { Grasse et généreuse (63) } \\
\text { Fermière } \\
\text { etc... }\end{array}$ & $\begin{array}{l}\text { Deuxième femme de } \\
\text { Mitien (141) } \\
\text { Maigre et mesquine (141) } \\
\text { Ménagère }\end{array}$ \\
\hline Actions & $\begin{array}{l}\text { Fait le voyage a Montréal } \\
\text { (122) } \\
\text { Fabrique le savon et s'affais- } \\
\text { se devant les cuves (145) } \\
\text { etc... }\end{array}$ & $\begin{array}{l}\text { Fait la vaissselle } \\
\text { et s'affaisse devant } \\
\text { l'évier (19) } \\
\text { Tue les petits chats de } \\
\text { Milien (51-52) }\end{array}$ \\
\hline
\end{tabular}

Si on met l'accent sur les différences (qualitications et actions) on peut définir deux personnages distincts. Mais ces contenus pourraient tout aussi bien être attribués à la mème personne. On sait que Milienne I a été très malade et que sa maladie l'a complètement transformée; rien n'empéche alors de voir dans la Milienne II la même personne mais transformée par la maladie, c'est l'interprétation que suggère par exemple le passage des pages 145-146. Cette assimilation est d'autant plus plausible que ces lemmes, en plus d'être désignées par le méme nom, partagent des contenus identiques (vieille + femme de Milien + morte d'une maladie intestinale). Cependant cette deuxième interprétation n'invalide pas la première. II semble impossible de lire ce récit d'une façon univoque. Le narrateur par le jeu homonymique entretient et exploite celte hésitation. Aucune instance ne vient dissiper l'ambiguité de l'histoire que le narrateur attribue à la conscience mensongère et incertaine du héros. On sait que Milien se raconte des histoires, qu'il ment:

L'imagination était une force mauvaise; elle faisait perdre le temps, elle constituait l'une des formes terribles de l'égarement. [...] elle le précipitait dans le monde des souvenirs, mais se souvenir n'était peut-étre que créer du présent? (p. 102).

C'était un pauvre vieux bonhomme s'aliénant dans de faux souvenirs. (143)

Ces interventions du narrateur qui déclare son héros menteur ou mythomane vient donc encore une fois vraisemblabiliser, naturaliser un procédé (l'homonymie) qui remet en cause la representation historique. 
Ces brouillages et ces bruits dans l'histoire de Milien sont aussi le fait des analepses. Dans te récit premier on peut assez facilement établir une version unique des faits comme nous avons tenté de le faire au début de cet essai; cependant cette reconstruction historique passe, comme nous l'avons vu aussi, par certains detours; le lecteur doit déjouer certains leurres qui sont mis sur sa route et qui ont pour effet de la confondre. L'histoire est rendue ambigué momentanément par l'effacement de certains procédés qui habituellement sont là pour produire l'illusion de cohérence. Comme l'écrit Jean Ficardou,

[...] dans un récit, le niveau réel et ce que nous pourrions nommer le niveau illusoire (l'onirique, le fantasmatique, I'hallucinatoire, l'imaginaire) ont un seul et mème statut.

Dans la mesure où ils sont tous, identiquement, de la fiction. Pour préserver, dans un récit, le réel de toute contamination, il faut donc prendre soin de le distinguer explicitement ${ }^{9}$.

Dans Les grands-pères plusieurs procédés sont utilisés pour distinguer le réel du rêve: formules déclaratives ("il songeait a ". "il se rappela ", "des images lui vinrent dans la tête ", etc.), indications temporelles, parenthèses, changements de temps, comparaisons, etc... Mais parfois ces transitions sont gommées, comme par exemple dans le chapitre premier ou dans le passage de la première visite à Chien Chien Pichlotte (p. 95-101) où les événements sont d'abord lus comme des faits réels et qui au cours de la lecture, rétrospectivement, apparaissent comme oniriques. L'ambiguilé du récit sur ce plan a donc un caractere provisoire et elle est mise presque toujours au compte de la focalisation interne.

Parmi les figures de transition, il faut compter aussi les laits de fréquence et de durée. La fréquence désigne le rapport de répétition qui existe entre le récit et l'histoire, entre l'événement raconté et l'ènoncé narratif (FIII, p. 145). Genelle distingue trois types de relations possibles selon que le récit raconte une fors ce qui s'est passé une fois (récit singulatif), plusieurs fois ce qui s'est passé une fois (récit répétitif), ou une seule fois ce qui s'est passé plusieurs tois (récit itératif). En reprenant le découpage antérieur, on peut se demander s'il y a changement dans la fréquence lorsque l'on passe du récit premier aux analepses.

A première vue on serait porté à dire que les segments du récit premier sont singulatifs et les segments rétrospectifs sont itératifs ou répétitifs : les analepses doivent être plus synthétiques étant donné qu'elles couvrent une période historique beaucoup plus longue que le récit premier ( 70 ans environ vs quelques heures). Or, on se rend compte que cette equation conventionnelle ou vraisemblable (récit premier: singulatif: récit second: itératif) ne s'applique pas aux Grands-pères. Si l'on s'en tient uniquement aux segments du recit premier, on remarque en effet une nette prédominance du singulatif (grammaticalement marqué par l'usage du passé simple). Même si à l'occasion on relève des passages itèratifs (6 passages itèratifs environ sur 23 segments), dans aucun de ces segments cet aspect ne l'emporte. Alors 
que dans les analepses on pourrait s'attendre à une fréquence différente, on relève à peu près la mème proportion: sur une trentaine de segments analeptiques, trois sont entierement itératifs, cinq font alterner litératif et le singulatif, et dans tous les autres cas (soit environ 22 segments sur 30) le singulatif domine. Donc il n'y a pas de différences marquées sur le plan de la fréquence entre le récit premier el les récits rétrospectifs.

L'absence d'écart ici est d'autant plus étonnant que, sur le plan de I'histoire, il y a un déséquilibre énorme entre l'amplitude chronologique de l'une et l'autre partie. Ce décalage (70 ans vs quelques heures) aurait dú avoir pour résultat de donner aux analepses un aspect beaucoup plus " ramassé " : "l'analepse prend le plus souvent la forme du récit sommaire" (FIII, p. 178). Or plutôt que de sommaires. qui ont recours avolontiers aux services de l'itératif "les analepses ici sont constituées par des scènes singulatives.

Comment interpréter l'usage de ce procédé chez Beaulieu? II semble que ce soit encore une technique utilisée pour favoriser l'équivoque. En effet l'usage d'une seule fréquence, qui efface la différence dans le mode de narration entre récit premier et analepse, facilite l'intégration et la coïncidence des niveaux narratifs du présent et du passé, du rêve et du réel. On relève un grand nombre de formules qui introduisent un épisode singulatif (comme "un jour", "un soir", "cette nuit-la") mais ces séquences ne sont jamais introduites dans la durée par un sommaire ou un segment itératif. Le sommaire et l'itératif ont une fonction généralisante qui permet de replacer tel événement isolé (singulatif) dans une durée. Cette transition sert de point de repere au lecteur classique habitué a la scansion scéne/sommaire. (Voir FIII, p. 170). Habituellement l'itératif marque un temps d'arrêt, un piétinement de l'histoire, il représente la stabilité dans la mobilité des événements, il permet au narrateur de faire le point, de proceder à la mise en place du décor et des faits, qui vont mettre en relief le fait singulatif et aménager l'enchaînement des scenes. Or ici rien de tel, l'absence relative de cet aspect chez Beaulieu explique la difficulté pour le lecteur de situer les événements les uns par rapport aux autres; ils sont toujours racontés comme des événements uniques sans jamais être datés ou replacés dans un contexte plus général. Nous passons d'une scène à une autre sans franchir l'étape transitoire. intermédiaire, de la phase itérative.

Nous avons vu comment dans Les grands-peres la construction analeptique et certains dispositifs d'ambiguité (homonymie, effacement des transitions) avaient une fonction antidiégétique et antireprésentative justifiant en quelque sorte les déclarations du romancier. Dans un article paru dans Perspectives, Beaulieu déclarait justement qu'il faisait de l'ambiguïté les bases de son travail d'ecrivain "1. Par contre ces procédés discursifs qui rendent le récit équivoque ne vont pas jusqu'à détruire complètement le caractère référentiel de la narration. L'extension de ces procédés est arrètée, endiguée par la diégèse première (donnée comme réelle) et par le code de focalisation (motivation psychologique), qui demeurent deux codes fondamentaux de lisibilité de ce texte. Le récit premier et les analepses qui 
désarticulent l'histoire, sont présentés comme le reflet d'un parcours réel (Milien errant dans son village natal) et d'une conscience en désordre. Cette double motivation maintient le texte dans le circuit de la vraisemblance.

Le récit des Grands-péres s'écrit dans cette tension constante entre un discours qui remet en question l'unité, la cohérence et la vérité de l'histoire et une nécessilé plus forte qui vient sans cesse justifier cette errance, ce désordre par le recours à une représentation réaliste. Nous avons souligné comment le discours du récit organise l'histoire, la détruit en partie, la rend équivoque et finalement la sert. La réussite de ce livre, son succés (attesté par le Prix littéraire de la ville de Montréal et une traduction anglaise) vient sans doute de cette tension entre le hasard el la nécessité, de ce compromis entre une écriture moderne, qui par le jeu d'un discours autotélique ébranle la lecture conventionnelle, et une esthétique qui préserve la fonction mimétique de la littèrature.

\section{Jacques MICHON Université de Sherbrooke}

1. "Ce brave soldat Chveik 1. Le Devoir (6 mars 1976). p. 14.

2. Seull, 1972, p. 67-282. Sera désigné par le sigle FIII.

3. Pour une définition de ces notions, outre Genelte. FIII, p. 71-74, on pourra consulter avec profit Guy Lafleche. Petit manuel des etudes titteraires. VLB éditeur, 1977. p. 43-44.

4. II s'agit d'un chiffre approximatif calcule à partir du nombre de lignes (en sachant qu'une page $=28$ lignes). Si le nombre total de pages ne correspond pas aux 146 pages effectives du texte, c'est qu'il faut soustraire de ce nombre les espaces blancs entre chaque chapitre et au début el à la fin du récit.

5. On a pu vérifier cette règle sur un corpus restreint d'ceuvres québécoises traditionnelles, alors que l'analyse de récits plus modernes (ex. les romans québecois publiés aprés 1960 ) indique déjà une transformation dans la distribution des anachronies. Voir a ce sujet la thèse de Pierre Hébert, Figures, temporalite et formes du discours narratit: essai de modèle et lectures de qualques couvres québécoises. Université Laval, 1977, I. 89. 152, 232.

6. Sur cette question de la substitution d'une durée objective par une durée subjective dans le récit. Voir J. Dubois et ali. Rhétorique générale, Larousse, 1970, p. 180.

7. L'analepse interne rapporte un épisode antérieur au point de l'histoire où l'on se trouve, mais postérieur au début du récit (FIII, p. 90). Par exemple dans Race de monde (chap. XI) Abel se rememore une conversation qu'il a eue la semaine prècèdente avec Steven.

8. Au contraire dans l'analepse complète la rètrospection vient se raccorder au récit premıer (FIII. p. 101). Ainsi dans Race de monde les chapitres III à VII racontent l'histoire des Beauchemin, qui a commencé avant le début du récit et s'achève à 
l'intérieur de ce dernier, là où il s'était arrêté pour faire place à celte rétrospection. Ainsi la boucle est bouclèe. Comme l'écrit Genette. ce type d'analepse "vise à récupérer la totalıté de l'antécédent narratif" (FIII, p. 101-102)

9. Le nouveau roman. "Ecrivains de toujours", Seuil, 1973, p. 91.

10. Pour une définitıon du sommaire el de la scéne, voir le chapitre de FIII sur la Duree, p. 130 et 141.

11. C'est à propos de la polysémie du nom de Saint-Jean-de-dieu là la fois maison psychiatrique et village natai) que Beaulieu déclarait : " Voici donc comment tout a commence entre Sarnt-Jean-de-Dieu et moi, par une ambiguité fondamentale dont j'ai fait ce qui me paraissait, du moins jusqu'a tout recemment, les bases de mon travail d'écrivain. "Adieu, Saint-Jean-de-Dieu". Perspectives, (22 mai 1976). p. 2. 